\title{
Clinical Reasoning: Transient speech deficits in a patient with history of medulloblastoma
}

Jessica D. Schulte, MD, PhD, Gunnar Hargus, MD, PhD, Peter Canoll, MD, PhD, Michael B. Sisti, MD, Tony J.C. Wang, Angela Lignelli, and Andrew B. Lassman, MD

Neurology ${ }^{\circledR}$ 2018;91:e1196-e1201. doi:10.1212/WNL.0000000000006202
Correspondence

Dr. Lassman

ABL7@cumc.columbia.edu

\section{Section 1: Case history}

A 58-year-old left-handed man presented with transient speech difficulty, blurry vision, and right arm heaviness.

The patient was initially diagnosed with a medulloblastoma (figure 1A) after posterior headaches led to brain MRI showing a right cerebellar mass. Molecular classification was not performed. After resection, the patient was treated with adjuvant cisplatin, amifostine, and craniospinal irradiation (3,600 cGy craniospinal with 1,800 cGy posterior fossa boost, $180 \mathrm{cGy}$ per fraction). Surveillance MRI scans performed multiple times per year showed no evidence of recurrence.

Six years after diagnosis, a surveillance MRI showed asymptomatic local recurrence. The patient underwent stereotactic radiosurgery (figure e-1, links.lww.com/WNL/A701) and high-dose chemotherapy with thiotepa, carmustine, and etoposide, followed by autologous stem cell transplant. Eight years after diagnosis, he reported 2 episodes of transient left homonymous hemianopsia. Imaging showed increased right cerebellar enhancement (figure 1, B.a and B.c). The patient underwent re-resection and pathology was consistent with radiation necrosis (figure 1B.a).

Twelve years after diagnosis, the patient began to have problems with speech, experiencing an episode of speech arrest lasting several minutes, and then a 30-minute episode of indiscernible speech. Over the next 3 years, he also developed dysarthria and problems with balance, coordination, and gait. Serial MRIs showed stable right cerebellar enhancement, considered to be residual radiation necrosis. Seventeen years after diagnosis, the patient experienced a 2-hour episode of difficulty with speech production and comprehension, right arm heaviness, and blurry vision. He was hospitalized, and neurologic examination at that time was normal except for mildly impaired speech fluency.

\section{Question for consideration:}

1. To where do the symptoms localize?

From the Departments of Neurology (J.D.S., A.B.L.), Pathology and Cell Biology (G.H., P.C.), Neurological Surgery (M.B.S.), Radiation Oncology (T.J.C.W.), and Radiology (A.L.), and the Herbert Irving Comprehensive Cancer Center (P.C., M.B.S., T.J.C.W., A.B.L.), Columbia University Irving Medical Center, New York, NY.

Go to Neurology.org/N for full disclosures. Funding information and disclosures deemed relevant by the authors, if any, are provided at the end of the article. 
Figure 1 Pathologic and radiologic findings
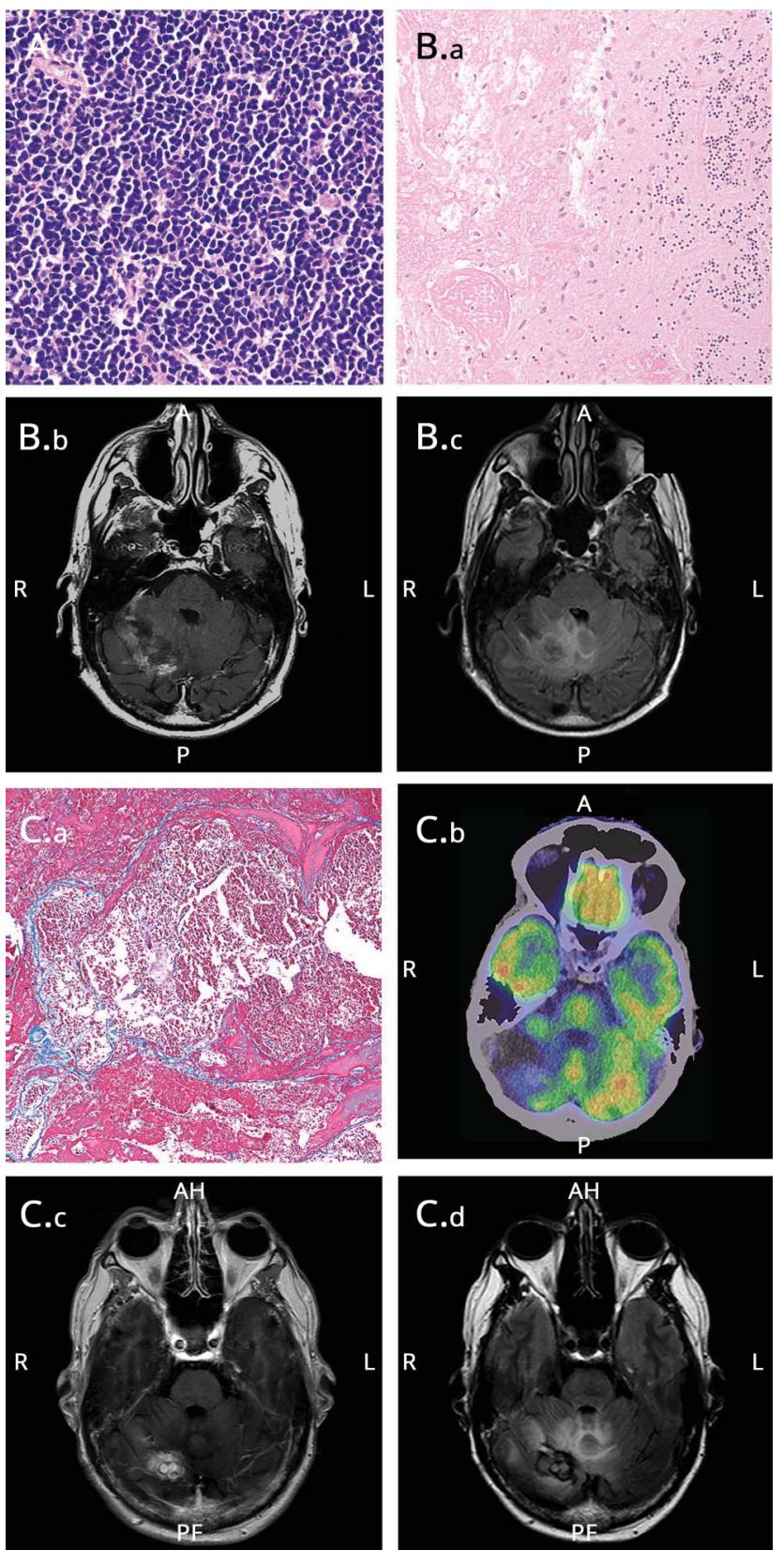

(A) Hematoxylin \& eosin (H\&E) stain of initial resection tissue demonstrates a highly cellular neoplasm arranged in sheets of lobules/clusters, infiltrating into the cerebellar parenchyma and subarachnoid space. The tumor cells demonstrate nuclear molding, high mitotic activity, and many apoptotic figures. Mixed foci express glial fibrillary acidic protein or synaptophysin, indicating glial and neuroblastic differentiation (not shown). (B.a-B.c) Pathology and MRI findings of radiation necrosis, resected 8 years after diagnosis. (B.a) H\&E stain demonstrates markedly thickened and hyalinized walls suggestive of radiation effects, on a background of necrosis with loss of granule and Purkinje cells. (B.b) MRI T1 postgadolinium sequence shows enhancement in the superior cerebellum. (B.c) MRI fluid-attenuated inversion recovery (FLAIR) sequence shows hyperintensity extending from the right cerebellar hemisphere into the vermis and right occipital lobe. (C.a-C.d) Pathology and MRI findings of cavernous malformation, resected 17 years after diagnosis. (C.a) Trichrome stain reveals large, dilated blood vessels, consistent with cavernous malformation, within a background of hemorrhagic, necrotic brain tissue. (C.b) ${ }^{18}$ F-FDG PET imaging shows hypometabolism in the right cerebellum. (C.c) MRI T1 post-gadolinium sequence, showing increased enhancement in the superior cerebellum extending into the right occipital lobe. (C.d) MRI FLAIR sequence shows the popcorn appearance found with some cavernous malformations, with FLAIR hyperintensity extending from the right cerebellar hemisphere into the vermis and right occipital lobe. FLAIR signal also extends into the right temporal lobe (not shown here). Susceptibility-weighted imaging by MRI also demonstrated low signal in the right cerebellum consistent with blood products (not shown).

GO TO SECTION 2 


\section{Section 2}

The patient's speech deficits may signify both motor and sensory aphasia, as well as dysarthria. Language requires integration of signals from several areas of the brain, including the frontal and temporal lobes, cerebellum, and thalamus. Motor aphasia typically localizes to the inferior frontal lobe, while sensory aphasia is associated with the posterior superior temporal lobe. Language is usually left-dominant, but in left-handed individuals (as this patient is), approximately $25 \%$ have right-dominant language. ${ }^{1}$ The cerebellum is implicated in several language functions, including motor coordination and syntax. Cerebellar lesions may result in ataxic dysarthria, speech apraxia, dysfluency, and less commonly, difficulty with naming, grammar, and comprehension. Cerebellar lesions may also cause aphasia via diaschisis from the contralateral frontal lobe. ${ }^{2}$
The description of right arm heaviness is nonspecific, and may represent weakness, numbness, or incoordination. A parsimonious localization for the speech and arm symptoms may be the left frontal lobe (contralateral weakness) or right cerebellum (ipsilateral coordination).

Finally, the episodic left homonymous hemianopsia localizes to any right-sided postchiasmatic structure, including the occipital lobe. Blurry vision suggests possible orbital involvement or increased intracranial pressure (ICP), although ICP more often causes diplopia and peripheral field defects.

\section{Question for consideration:}

1. What are the etiologies of transient neurologic deficits in patients with brain cancer? 


\section{Section 3}

\section{Seizures}

Tumors can produce seizures through many mechanisms, including mass effect, edema, hemorrhage, ischemia, hydrocephalus, and alterations in tumor microenvironment that induce neuronal hyperexcitability. Primary brain malignancies carry a higher seizure risk than metastases. Seizures in medulloblastoma are less frequent than in glioma, possibly related to the posterior fossa being less epileptogenic. ${ }^{3}$ However, a recent study reported that $8 \%$ of children with medulloblastoma develop seizures. ${ }^{3}$ Psychogenic/nonepileptic seizures may also present with focal features.

\section{Cerebrovascular disease}

The prothrombotic state of cancer increases risk for TIA and arterial strokes, as well as sinus venous thrombosis and resultant venous infarct. Solid tumors can also cause local ischemia through mechanical compression of intracranial blood vessels. Radiation therapy (RT) can cause vascular fibrosis and stenosis. ${ }^{4}$

\section{ICP plateau waves}

ICP plateau waves, first described by Nils Lundberg in $1960,{ }^{5}$ are transient pathologic increases in ICP that can manifest as headache, blurry vision, confusion, hemiparesis, aphasia, and other focal neurologic deficits. Episodes typically last 2-30 minutes and can be triggered by positional changes or noxious stimuli. Plateau waves are caused by a variety of neurologic conditions that impair CSF absorption, including traumatic brain injury, stroke, tumors, and leptomeningeal disease. ${ }^{5}$

\section{Stroke-like migraine attacks after radiation therapy}

Stroke-like migraine attacks after radiation therapy (SMART) syndrome is rare, affecting patients years after radiation. Patients develop focal neurologic deficits with or without headache, and cortical enhancement on MRI, which all resolves over the course of weeks with corticosteroid treatment. The pathophysiology may involve dysfunction in cortical metabolism and vasculature permeability., ${ }^{4,6}$ Complex migraine and migraine with aura can also produce transient focal neurologic symptoms.

\section{Question for consideration:}

1. What mechanisms could trigger the transient phenomena in this patient, and what tests should be ordered? 


\section{Section 4}

Oncologic causes of transient neurologic deficits.

\section{Cancer recurrence}

In a retrospective study of adult medulloblastoma, 74 of 238 patients $(31.1 \%)$ had recurrent disease, with one-third located in the posterior fossa, and the remainder found supratentorially, in the spine, or outside the CNS. ${ }^{7}$ Recurrence is typically in the first 2 years in children, and 2-7 years after diagnosis in adults. ${ }^{7}$ Recurrence could induce focal deficits, seizures, edema, or infarcts via mass effect. Intratumoral hemorrhage is rare with medulloblastoma.

\section{Leptomeningeal metastases}

Leptomeningeal spread is common in medulloblastoma, and present to varying degrees in other primary and secondary brain malignancies. Leptomeningeal metastases (LM) can cause cranial neuropathies, as well as focal deficits and seizures through superficial invasion of cortex. LM can also obstruct leptomeningeal veins and arteries, predisposing to ICP plateau waves and tissue ischemia. ${ }^{4} \mathrm{LM}$ can be occult on MRI even with contrast, and when suspected, a high-volume lumbar puncture should be performed with cytology evaluated along with opening pressure, routine chemistry, and cell counts.

\section{Radiation-induced brain pathology}

RT can alter brain vasculature in many ways, including vessel wall fibrosis, luminal stenosis, and resultant ischemia. Longterm complications include radiation necrosis, ${ }^{8}$ as seen in this patient, secondary malignancies such as new brain cancer, and vascular malformations. Cavernous malformations can cause focal deficits and seizures through mass effect, edema, and hemorrhage. Seizures are the most common presenting symptom of cavernous malformations. ${ }^{6}$

\section{Evaluation}

Symptoms did not recur during hospitalization. EEG showed bitemporal slowing, but no epileptiform discharges. MRI demonstrated right cerebellar enhancement, microhemorrhage, and fluid-attenuated inversion recovery (FLAIR) hyperintensity extending into the vermis, right temporal, and occipital lobes. MRI spine showed subtle enhancement of the cord surface at T7, possibly representing leptomeningeal disease, vs motion artifact. Magnetic resonance (MR) angiography was unremarkable. MR spectroscopy was inconclusive due to motion artifact. MR perfusion performed 2 weeks prior to planned resection showed increased blood flow, while MR perfusion performed the day of resection did not show increased blood flow. Brain ${ }^{18} \mathrm{~F}$ FDG PET showed decreased metabolism in the right cerebellum (figure 1C.b), although changes in longitudinal PET scanning can assist in distinguishing inflammatory or infectious processes from tumor recurrence. Lumbar puncture showed an opening pressure of $16.5 \mathrm{~cm} \mathrm{H}_{2} \mathrm{O}$, no white blood cells, protein 60, glucose 67 (serum glucose 92), and negative cytology.

\section{Course}

The patient underwent resection, and pathology was consistent with cavernous malformation (figure 1C.a). After resection, symptoms initially resolved, although 9 months later, the patient had an episode of transient left-sided homonymous scotoma, accompanied by left arm and mouth numbness. He continues to have transient episodes of garbled speech and right hand numbness and tingling. MRIs have remained stable. It is suspected that these symptoms may represent recurrent seizures or transient ischemia due to RT induced vasculopathy.

\section{Discussion}

A 58-year-old man with history of recurrent right cerebellar medulloblastoma presented with transient aphasia, right arm heaviness, and blurry vision. He was found to have a right cerebellar cavernous malformation, likely caused by prior high-dose radiation. Both spontaneous and RT-induced cavernomas can cause seizures. A complex partial seizure with electrographic field involving the cerebellum and adjacent temporal and occipital lobes provides a unifying explanation for the patient's symptoms. Further supporting this localization is improvement in his symptoms after resection of the cavernoma.

Treatment of medulloblastoma in adults historically involved surgery, radiotherapy, and often chemotherapy, ${ }^{7}$ although treatment is evolving as understanding of the underlying molecular biology ${ }^{9}$ improves.

Radiation causes acute and chronic effects to brain vasculature. While vasogenic edema occurs days after treatment, chronic effects may not manifest until months to years later. In addition to chronic vascular fibrosis, radiation can induce endothelial mutagenesis and aberrant vascular endothelial growth factor signaling that can trigger formation of vascular malformations. ${ }^{6}$ In children, medulloblastoma is the brain tumor most often associated with RT-induced vascular malformations. ${ }^{6}$ MRI may show a popcorn appearance on T2/FLAIR, although other treatment effects may obscure this finding. While functional modalities (MR spectroscopy, MR perfusion, PET) may be helpful in distinguishing tumor recurrence from radiation necrosis, ${ }^{8}$ the role of functional imaging in diagnosing cavernomas is not well-described.

While more common in children, RT-associated cavernous malformations are also documented in adults who received large doses of RT (>3,000 cGy), as seen with our patient. ${ }^{6,10}$ Higher doses of RT also correlate with shorter latency to development of these malformations. ${ }^{10}$ Presentation and symptoms vary. In one case series, $50 \%$ of adult patients were asymptomatic, and the remaining patients presented with headaches (19\%), seizures (19\%), hemorrhage (13\%), and focal deficits (13\%). ${ }^{10}$ Lesions may be clinically monitored, 
but surgery is often considered for diagnostic or therapeutic purposes. $^{6}$

\section{Author contributions}

Jessica D. Schulte: review of clinical information, design of figure, author of manuscript. Gunnar Hargus: imaging and description of pathology samples. Peter Canoll: review of pathology imaging, critical revision of content for intellectual content. Michael Sisti: review of clinical information, critical revision of content for intellectual content. Tony J.C. Wang: review of clinical information, review and provision of radiotherapy planning and figures. Angela Lignelli: review of clinical information, review of images, review of figures. Andrew B. Lassman: project supervision, critical revision of content for intellectual content.

\section{Study funding}

No targeted funding reported. A. Lassman was supported in part by 5P30CA013696-43 and 5UG1CA189960-04 (unrelated to the current study).

\section{Disclosure}

J. Schulte, G. Hargus, P. Canoll, and M. Sisti report no disclosures relevant to the manuscript. T. Wang reports personal fees and nonfinancial support from AbbVie, nonfinancial support from Merck, personal fees from AstraZeneca, personal fees from Doximity, nonfinancial support from
Novocure, personal fees and nonfinancial support from Elekta, and personal fees from Wolters Kluwer, outside the submitted work. A. Lignelli reports no relevant disclosures regarding the submitted work. A. Lassman reports honoraria from prIME Oncology, WebMD, Italian Association for Cancer Research, American Society of Clinical Oncology, Bioclinica, Celgene, Sapience, AbbVie, Cortice, Kadmon, Novocure, and AstraZeneca, all outside the submitted work. Go to Neurology.org/N for full disclosures.

\section{References}

1. Knecht S, Dräger B, Deppe M, et al. Handedness and hemispheric language dominance in healthy humans. Brain 2000;123:2512-2518.

2. Marien $P$, Beaton A. The enigmatic linguistic cerebellum: clinical relevance and unanswered questions on nonmotor speech and language deficits in cerebellar disorders. Cerebell Ataxias 2014;1:12.

3. Ullrich NJ, Pomeroy SL, Kapur K, Manley PE, Goumnerova LC, Loddenkemper T. Incidence, risk factors, and longitudinal outcome of seizures in long-term survivors of pediatric brain tumors. Epilepsia 2015;56:1599-1604.

4. DeAngelis LM, Posner JB. Neurological Complications of Cancer. New York: Oxford University Press; 2008.

5. Masayuki M, Shunichi Y, Hajime H, Hiroshi G. Cerebral hemodynamic changes during plateau waves in brain-tumor patients. J Neurosurg 1979;50:483-488.

6. Morris B, Partap S, Yeom K, Gibbs IC, Fisher PG, King AA. Cerebrovascular disease in childhood cancer survivors: a Children's Oncology Group Report. Neurology 2009; 73:1906-1913.

7. Padovani L, Sunyach M-P, Perol D, et al. Common strategy for adult and pediatric medulloblastoma: a multicenter series of 253 adults. Int J Radiat Oncol Biol Phys 2007;68:433-440.

8. Verma N, Cowperthwaite MC, Burnett MG, Markey MK. Differentiating tumor recurrence from treatment necrosis: a review of neuro-oncologic imaging strategies. Neuro Oncol 2013;15:515-534.

9. Remke M, Hielscher T, Northcott PA, et al. Adult medulloblastoma comprises three major molecular variants. J Clin Oncol 2011;29:2717-2723.

10. Heckl S, Aschoff A, Kunze S. Radiation-induced cavernous hemangiomas of the brain. Cancer 2002;94:3285-3291. 


\title{
Neurology
}

\author{
Clinical Reasoning: Transient speech deficits in a patient with history of \\ medulloblastoma \\ Jessica D. Schulte, Gunnar Hargus, Peter Canoll, et al. \\ Neurology 2018;91; $1196-\mathrm{e} 1201$ \\ DOI 10.1212/WNL.0000000000006202
}

This information is current as of September 17, 2018

\section{Updated Information \& Services \\ References \\ Citations \\ Subspecialty Collections}

Errata

Permissions \& Licensing

Reprints including high resolution figures, can be found at: http://n.neurology.org/content/91/12/e1196.full

This article cites 9 articles, 2 of which you can access for free at: http://n.neurology.org/content/91/12/e1196.full\#ref-list-1

This article has been cited by 1 HighWire-hosted articles: http://n.neurology.org/content/91/12/e1196.full\#\#otherarticles

This article, along with others on similar topics, appears in the following collection(s):

Aphasia

http://n.neurology.org/cgi/collection/aphasia

Cerebellum

http://n.neurology.org/cgi/collection/cerebellum

Primary brain tumor

http://n.neurology.org/cgi/collection/primary_brain_tumor Radiation therapy-tumor

http://n.neurology.org/cgi/collection/radiation_therapytumor

An erratum has been published regarding this article. Please see next page or:

/content/91/22/1031.1.full.pdf

Information about reproducing this article in parts (figures,tables) or in its entirety can be found online at:

http://www.neurology.org/about/about_the_journal\#permissions

Information about ordering reprints can be found online:

http://n.neurology.org/subscribers/advertise

Neurology ${ }^{\circledR}$ is the official journal of the American Academy of Neurology. Published continuously since 1951, it is now a weekly with 48 issues per year. Copyright @ 2018 American Academy of Neurology. All rights reserved. Print ISSN: 0028-3878. Online ISSN: 1526-632X.

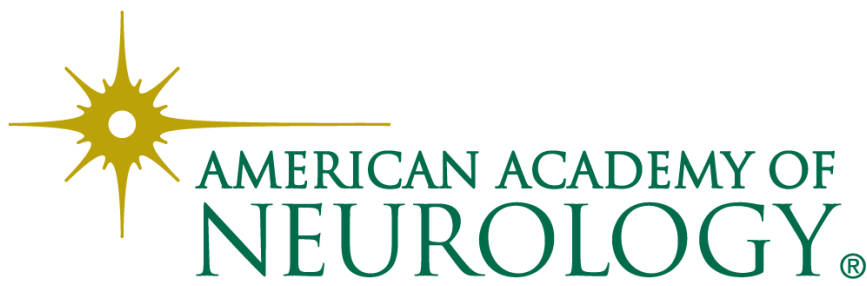




\title{
Disputes \& Debates: Editors' Choice
}

Steven Galetta, MD, FAAN, Section Editor

\begin{abstract}
Editors' note: Demoralization in Parkinson disease
In the article "Demoralization in Parkinson disease," Koo et al. reported that demoralization, with a prominent inability to cope, was common in their sample of 94 participants with Parkinson disease (PD), and that it was associated with motor dysfunction. In response, Bruno et al. report the findings of an exploratory study in patients with advanced PD, which found that while demoralization and depression tended to coexist, demoralization was correlated with PD severity, whereas depression increased caregiver strain, further suggesting that these are different constructs. In reply, author Koo agrees that their findings are complementary, and discusses their favored conceptualization of depression vs demoralization, suggesting that social support and perceived social support may safeguard against demoralization.
\end{abstract}

Aravind Ganesh, MD, and Steven Galetta, MD

Neurology ${ }^{\circledR}$ 2018;91:1029. doi:10.1212/WNL.0000000000006566

\section{Reader response: Demoralization in Parkinson disease}

Veronica Bruno (Calgary, Canada), Deborah Mancini (Toronto, Canada), Mateusz Zurowski (Toronto, Canada), and Janis M. Miyasaki (Edmonton, Canada)

Neurology ${ }^{\circledR}$ 2018;91:1029. doi:10.1212/WNL.0000000000006568

We read with interest the article by Koo et al. ${ }^{1}$ and agree with the authors about the relevance of demoralization in Parkinson disease (PD). Adding to their results, we performed an exploratory study in a movement disorders palliative care clinic on demoralization and depression in patients with advanced PD ( $\geq 2.5$ on the Hoehn \& Yahr scale) and their relationship with caregiver burden. Patients were assessed using the Beck Hopelessness Scale (BHS), the Beck Depression Inventory (BDI), the caregivers Zarit Burden Interview, as well as scales for anxiety, quality of life, and motor symptoms.

In our population $(n=43), 100 \%$ of the demoralized patients were depressed. Ten patients had depression; of those, $70 \%$ were demoralized. Linear regression models adjusting for age and sex showed that BDI scores strongly correlated with BHS $(\beta=0.98, p=0.002)$ and with the caregiver burden $(\beta=0.42, p=0.008)$. The BHS score correlated with the Movement Disorders Society-sponsored revision of the Unified Parkinson's Disease Rating Scale III (MDS-UPDRS III) $(\beta=0.15, p=0.04)$.

Our results support those by Koo et al.: demoralization and depression are different constructs. Demoralization seems to correlate to MDS-UPDRS III, but not depression. Depression, but not demoralization, appears to increase caregiver strain. Therefore, differentiating demoralization from depression may be important because of the possibility of different therapeutic approaches, even for patients with advanced disease.

1. Koo BB, Chow CA, Shah DR, et al. Demoralization in Parkinson disease. Neurology 2018;90:e1613-e1617.

Copyright (C) 2018 American Academy of Neurology

Author disclosures are available upon request (journal@neurology.org). 


\section{Author response: Demoralization in Parkinson disease}

Brian B. Koo (Orange, CT)

Neurology ${ }^{\circledR}$ 2018;91:1030. doi:10.1212/WNL.0000000000006569

I thank Bruno et al. for their comment on demoralization in Parkinson disease (PD). Certainly, it makes sense to explore feelings of hopelessness when studying demoralization, as hopelessness is more specific to demoralization than is depressed mood. I am curious if Bruno et al. found this to be the case in their PD cohort. It is interesting that their findings mirror ours, ${ }^{1}$ in that demoralization was associated with the Movement Disorders Society-sponsored revision of the Unified Parkinson's Disease Rating Scale III. The finding that depression, but not demoralization, is associated with caregiver burden is interesting and also makes sense. Demoralization and depression are overlapping yet distinct feeling states. Demoralization is characterized by subjective incompetence and a loss of a cognitive map, but willpower remains. In depression without demoralization, the cognitive map is intact but willpower is gone. Social support and perceived social support are also important factors that safeguard against demoralization. I commend Bruno et al. for looking at the important issue of demoralization in PD and for having the foresight to include an assessment of the caregiver and his or her burden.

1. Koo BB, Chow CA, Shah DR, et al. Demoralization in Parkinson disease. Neurology 2018;90:e1613-e1617.

Copyright @ 2018 American Academy of Neurology

\section{Editors' note: Teaching Neurolmages: Multimodality imaging of carotid web}

In "Teaching NeuroImages: Multimodality imaging of carotid web," Renard et al. presented representative images of a carotid web identified in a 52-year-old woman presenting with an ipsilateral acute middle cerebral artery infarction on 3 modalities: Doppler ultrasound, CT, and magnetic resonance angiography. In response, Dr. Sharma discusses the relative strengths and limitations of different modalities in identifying carotid webs. Dr. Sharma notes that in an ongoing study of high-resolution MRI in patients with significant symptomatic carotid stenosis, carotid webs were identified in about $22 \%$ of cases, but that the role of such webs in cryptogenic stroke remains unknown.

Aravind Ganesh, MD, and Steven Galetta, MD

Neurology ${ }^{\circledR}$ 2018;91:1030. doi:10.1212/WNL.0000000000006570

\section{Reader response: Teaching NeuroImages: Multimodality imaging of carotid web}

Vijay K. Sharma (Singapore)

Neurology ${ }^{\circledR}$ 2018;91:1030-1031. doi:10.1212/WNL.0000000000006571

I read with interest the ultrasound and CT angiographic (CTA) findings of carotid web. ${ }^{1}$ This entity is recognized more often, probably due to improved resolution of various diagnostic modalities. Duplex ultrasonography and CTA can identify large webs, especially when associated with secondary mild calcification or a superimposed thrombus. Presence of larger amount of calcium interferes with the spatial resolution of CTA. ${ }^{2}$ These limitations may be 
overcome by high-resolution MRI (HR-MRI) of the carotid wall. HR-MRI enables visualization of the webs that are small, thin, membranous, filamentous, or diaphragm-like. ${ }^{3,4} \mathrm{~A}$ recent large acute stroke trial reported carotid web on the symptomatic side in $2.5 \%$ of patients. ${ }^{5}$ In an ongoing prospective study of HR-MRI in patients with $>50 \%$ symptomatic carotid stenosis, we observed carotid webs of various morphologies in about $22 \%$. Various protocols may help with in-depth evaluation of carotid web morphology, composition, as well as associated hemodynamic alterations. Whether carotid webs play an etiopathogenic role in cryptogenic stroke remains unknown. This important question can be answered only by larger prospective studies with uniform imaging protocols.

1. Renard D, Hampton J, Keita M, Freitag C. Teaching NeuroImages: multimodality imaging of carotid web. Neurology 2018;90:e1541.

2. Bishop PD, Feiten LE, Ouriel K, et al. Arterial calcification increases in distal arteries in patients with peripheral arterial disease. Ann Vasc Surg 2008;22:799-805.

3. Cappendijk VC, Cleutjens KB, Kessels AG, et al. Assessment of human atherosclerotic carotid plaque components with multisequence MR imaging: initial experience. Radiology 2005;234:487-492.

4. Boesen ME, Eswaradass PV, Singh D, et al. MR imaging of carotid webs. Neuroradiology 2017;59:361-365.

5. Compagne KCJ, van Es ACGM, Berkhemer OA, et al. Prevalence of carotid web in patients with acute intracranial stroke due to intracranial large vessel occlusion. Radiology 2018;286:1000-1007.

Copyright (c) 2018 American Academy of Neurology

\section{CORRECTIONS}

\section{Clinical Reasoning: Transient speech deficits in a patient with history of medulloblastoma}

Neurology ${ }^{\circledR} 2018 ; 91: 1031$. doi:10.1212/WNL.0000000000006706

In the Clinical Reasoning piece "Transient speech deficits in a patient with history of medulloblastoma” by Schulte et al., ${ }^{1}$ the byline is missing degrees for two authors. The author list should have included “Tony J.C. Wang, MD” and “Angela Lignelli, MD.” The authors regret the error.

\section{Reference}

1. Schulte JD, Hargus G, Canoll P, et al. Clinical Reasoning: Transient speech deficits in a patient with history of medulloblastoma. Neurology 2018;91:e1196-e1201.

\section{Financial relationships between neurologists and industry: The 2015 Open Payments database}

Neurology ${ }^{\circledR} 2018 ; 91: 1031$. doi:10.1212/WNL.0000000000006707

In the Contemporary Issues piece "Financial relationships between neurologists and industry: The 2015 Open Payments database" by Ahlawat and Narayanaswami, ${ }^{1}$ there is an error at the top of the second column on page 1065, which should state "The payments to these 6 neurologists totaled $\$ 259,858$ " rather than "The payments to these 6 neurologists totaled $\$ 2,59,858$." The authors regret the error.

\section{Reference}

1. Ahlawat A, Narayanaswami P. Financial relationships between neurologists and industry: the 2015 Open Payments database. Neurology 2018;90:1063-1070. 